\title{
Optical Glass of Interferometer and Schlieren Quality for Wind-Tunnel Optics
}

\author{
By Leroy W. Tilton
}

\begin{abstract}
Large disks for wind tunnels should be uniform in optical thickness within $\pm 1 / 16 \lambda$ if highest accuracy in measurements by interference fringes is to be insured. This means that heterogeneities in whe glass shall be less than $\pm 5 \times 10^{-7}$ in refractive index for thicknesses of 3 or 4 centimeters. Evidence is cited to show that differences in chemical composition in good glass may not prevent realization of the desired uniformity, and that stress birefringence cannot be an important preventing factor. The remaining source of nonuniformity in glass is the existence of temperature gradients during annealing that can cause changes in structure, and it has been found that these can be reduced within required limits simply by encasing the glass, during annealings, in a sufficient number of concentric boxes composed of alternately heat-conducting and insulating layers.
\end{abstract}

\section{Introduction}

Demands for large interferometer plates (beam splitters) and large windows for schlieren apparatus have occasioned renewed interest in the existing degree of uniformity of optical glass and in possible improvements. Because of imperfections in glass, the figuring of optical surfaces has often been employed, especially when linear apertures are to be large as in the larger astronomical objectives, and when the optical path in the glass must be long as is the case in some large prisms. Figuring procedures were sometimes found necessary, because the optical performance was otherwise poor and unacceptable.

Figuring, or local repolishing, of optical surfaces is done only by expert artisans, perhaps artists in a way, and as we progress in modern methods of the design and construction of optical systems, it becomes increasingly difficult to find experienced men who can undertake figuring with confidence. Consequently the glass makers have been and are improving the quality of their product, but they cannot be expected to do so in advance of widespread demands for glass of better quality.

The quality of optical glass is commonly judged by its freedom from color, seeds, stones, feather, striae, and other plainly visible defects and also by its freedom from internal stresses as indicated by tests for birefringence. Usually, glass that is rated high in these respects has been found by the tests of actual use to be satisfactory in many precise optical instruments, and it has been generally believed that exceptions were caused by inherent nonuniformity in chemical composition. The work of Tool $[1]^{1}$ and his associates at this Bureau has shown, however, that glass may be physically inhomogeneous, because of temperature gradients that existed during heat treatment, and still show little or no stress birefringence and be quite uniform in chemical composition. As a result some previous failures can be explained, it is possible to successfully retreat and recondition some elements that have failed in optical performance, and best of all steps can be indicated that will lead to much better uniformity in the refractivity of glass.

For some purposes, refractive uniformity may be desirable for a given type of glass that is produced over long periods, as the successive pots are made, or it may be necessary merely that many small pieces from a given pot of glass shall be equally refractive. In these cases, there is no problem in testing the degree of the existing uniformity that is attained. In single large pieces of

\footnotetext{
1 Figures in brackets indicate the literature references at the end of this paper.
} 
glass, however, we have no completely satisfactory way of knowing just what degree of refractive uniformity exists. Optical homogeneity is not assured by freedom from birefringence and visible defects. Interference methods of investigating homogeneity become very difficult when the thickness of glass exceeds 2 or $2 \frac{1}{2}$ in., because the fringes formed by reflections from the surfaces involve such a large difference in equivalent air path, namely $2 n t$, that they are indistinct and have seldom or never been accurately and quantitatively observed. Prism methods of measuring the differences in refractivity require depletion or destruction of the blanks, or else yield merely some sort of an average value for long paths in the glass.

\section{Permissible Phase Differences}

Strict tolerances in uniformity of optical glass become progressively more important as activities in the optical industry become less a matter of individual skill and thus approach the American ideal of standardization for mass production. In general, the designers' tolerances, whether concerned with the usual instruments for taking precision observations or with the exacting requirements of certain fixed-focus instruments, are comparatively liberal. They relate chiefly to intermelt and interblank uniformity rather than to the intrablank homogeneity that is of importance in wind-tunnel optics.

In setting tolerances in optical uniformity, it is generally assumed that heterogeneity of refracting media of optical systems can be tolerated unless a noticeable amount of aberration is introduced, that is, an amount which results in something short of "best definition" as judged, usually, by the observation of the diffraction pattern of a "point" source. For this limiting condition, Rayleigh [2] placed the limiting departure from true sphericity of wave front at $1 / 4-\lambda$ phase difference. At this limit the "aberration begins to be decidedly prejudicial." But Rayleigh considered only certain types of aberration, and it is possible that other types might necessitate a less liberal tolerance. Very little is known precisely concerning the actual defects in imagery that may be produced by irregular refractivity in lens systems.

Chalmers [3] found that in practice the figuring of small objectives is employed to reduce path differences to $1 / 5 \lambda( \pm 1 / 10 \lambda$ from the mean $)$ or less to meet the requirements of definition. As a result of an experimental study of the distribution of intensities near a focus, Martin [4] concludes that "to secure the placing of the full amount of light into its correct place in the image such phase residuals should be brought within $1 / 6 \lambda$ at the best visual focus". This is fairly in accord with the suggestion of Conrady [5] that "for instruments of the highest quality the limits should be reduced to $1 / 6$ or even $1 / 8$ of a wave-length", because with the classical Rayleigh limit, "there is in every case a decided loss of contrast in the image of an extended object". Elsewhere [6] the same author has considered the requirements for depth of focus and focal range without serious loss of definition. He concludes that it is desirable to keep the residuals of aberration well below the limit set by the requirements of definition and contrast at any one definite focus. For maximum focal range, zero phase difference would be required, but values of $1 / 8$ and $1 / 16 \lambda$ are mentioned by Conrady as limits of phase difference at which the discrepancies between maximum and realized focal range are "unimportant" and "quite insensible", respectively. This is of importance in microscopy with high numerical apertures, especially for projection and photographic methods.

So far, only resolution and contrast have been implicitly considered in relation to definition. Wadsworth, [7] however, has emphasized the importance of accuracy as an element of good definition (using the latter term in the broad sense), which is often of more importance than either resolution or contrast. He finds the requirements for "metrological power" more exacting than those for either resolving or delineating power. For instruments intended for maximum accuracy of measurement, he concludes that the differential distortion of those wave fronts that form simultaneous or successive images on which settings are to be made must not exceed one-fourth of that which would affect good optical definition according to Rayleigh. That is, Wadsworth places the phase difference limit at $1 / 16 \lambda$ instead of $1 / 4 \lambda$ as did Rayleigh. It is owing to slight shifts of the distribution of energy in the diffraction pattern that error can arise from such small aberration, and if the effect is constant between successive measurements, then a difference of such measurements, which 
frequently is all that is desired, is of course free from such error. But after an examination of the conditions under which such small aberrations are constant, Wadsworth decides that it is impossible in all cases to secure such conditions and that the only entirely safe procedure is to reduce the phase differences themselves to the small value of $1 / 16 \lambda$. This, probably, is the desirable phase tolerance in some applications of wind-tunnel opties.

\section{Effects of the Distribution of Heterogeneity}

In order to decide upon the degree of heterogeneity permissible without violation of a given degree of phase uniformity, it is necessary to consider the distribution of the departure from the average optical density of the refracting media. Some useful ideas, directly applicable to a consideration of wind-tunnel optics, are obtained from an examination of a few simple cases:

(1) For any optical component having plane surfaces (i. e., producing zero effect on the vergence of transmitted light), a small uniform transverse optical density gradient will introduce no appreciable aberration but merely a deviation in the direction of propagation of the wave.

(2) For any optical component, a more or less concentric and systematically progressive index gradient from center to periphery may produce chiefly longitudinal and symmetrical aberration that can usually be partially eliminated by refocusing of the viewing system.

(3) If the distribution of abnormal density is somewhat irregular it is quite possible that, of the total aperture, certain moderately large ${ }^{2}$ areas are covered with glass of different average index from that of other large areas. In some such cases the result can be almost entirely one of asymmetrical aberration, the amount being the maximum that can result from the index deviations present.

Even in case (3), however, it may happen that some hypothetical regular transverse index gradient from center to edge can approximate the

\footnotetext{
${ }^{2}$ It is necessary to distinguish this case from that which usually obtains when striae or other local abrupt changes in density are present. (See A. Arnulf, Rev. D'Optique 6, 2 (1927). In glass of good quality these can affect only small proportions of the total apertures, unless the latter a re themselves small, as in microscope objectives. Consequently, the intensity of the out-of-phase light due to striae is in general so small a proportion of the total that the noticeable effects are of a much smaller order than those to be referred to in this discussion.
}

existing nonuniformities and thus permit amelioration of the irregular effects by simple orientation and refocusing.

\section{An Index-Heterogeneity Tolerance}

In any event, it is useful to establish the indexheterogeneity tolerance corresponding to the residual effects referred to under case (3). If two rays traverse paths of equal length, $a$, through glasses of indices $n^{\prime}$ and $n^{\prime \prime}$, respectively, the numbers of wavelengths are

$$
N^{\prime}=\frac{a n^{\prime}}{\lambda},
$$

and

$$
N^{\prime \prime}==\frac{a n^{\prime \prime}}{\lambda},
$$

whence the optical path difference is

$$
\left(N^{\prime \prime}-N^{\prime}\right)=\frac{a\left(n^{\prime \prime}-n^{\prime}\right)}{\lambda},
$$

where $\lambda$ is the wave length in air. If $\left(n^{\prime \prime}-n^{\prime}\right)$ be expressed as $2 \Delta n, n$ being the average index of the glasses, and $\left(N^{\prime \prime}-N^{\prime}\right)$ be expressed as $2 \Delta N$, where $\pm \Delta n$ and $\pm \Delta N$ are deviations from the mean values of $n$ and $N$, then for any given tolerance in phase, $2 \Delta N$ (maximum phase difference), the corresponding tolerance in index (departure from the mean) is expressible as

$$
\Delta n= \pm \frac{\Delta N \lambda}{a}
$$

For a glass thickness of $1 \mathrm{~cm}$, table 1 shows the corresponding values of $\Delta n$ computed according to the Rayleigh and also to the Wadsworth limit for a few frequently used wavelengths.

\begin{tabular}{|c|c|c|c|c|c|}
\hline \multirow{2}{*}{$\begin{array}{l}\text { Phase } \\
\text { differ- } \\
\text { ence, } \\
2 \Delta N\end{array}$} & \multicolumn{5}{|c|}{ Tolerances in refractive index, $\Delta n$} \\
\hline & $\begin{array}{c}C \\
6,563 A\end{array}$ & $\begin{array}{c}D \\
5,893 A\end{array}$ & $\begin{array}{c}F \\
4,861 \mathrm{~A}\end{array}$ & $\stackrel{g}{4,358 \mathrm{~A}}$ & $\begin{array}{c}h \\
4,047 \mathrm{~A}\end{array}$ \\
\hline $1 / 4 \ldots \ldots$ & $\begin{array}{l} \pm 0.000008 \\
\pm .0000021\end{array}$ & $\begin{array}{l} \pm 0.000007 \\
\pm .0000018\end{array}$ & $\begin{array}{l} \pm 0.000006 \\
\pm .0000015\end{array}$ & $\begin{array}{l} \pm 0.000005 \\
\pm .0000014\end{array}$ & $\begin{array}{l} \pm 0.000005 \\
\pm .0000013\end{array}$ \\
\hline
\end{tabular}

TABLE 1. Suggested maximum permissible phase differences and corresponding homogeneity tolerances in $\Delta n$ for $1-\mathrm{cm}$ glass path

Considering, on one hand, the smallness of these index differences in comparison with those known to exist in many glasses, and, on the other, the known degree of perfection attained in many optical systems, sharp disparity appears, but 
several considerations may be mentioned to show that no inconsistency necessarily exists.

In the first place the tolerances correspond only to case (3), that of the most unfavorable of the possible distributions of index heterogeneity. Secondly, excellence of performance is quite frequently judged by the relatively easily attained standard of theoretical resolving power, based on $1 / 4-\lambda$ phase difference. Even a phase difference as large as $1 / 2 \lambda$ does not always entirely prevent resolution [8]. Furthermore, the really exacting tests, involving the accuracy of measurements carried out under conditions requiring constancy of aberration are seldom made. Wadsworth treated some cases of this kind in detail. The heliometer and the spectrograph are examples where varying portions of the apertures of the optical components come into use for the different measurements. Varying angular presentation of the wave front to the optical surfaces is one of the most commonly encountered conditions of a nature giving opportunity for exact tests. This occurs whenever the telescope is used for measurements on images having considerable angular separation in the field of view and in the use of cameras for airplane mapping. But in most such cases, even the errors of design are not reduced to negligible proportions, and exact tests are not made because the calibration is relied upon to include also the errors of material and workmanship, or because null methods are used to eliminate all error, as in the test for the Einstein effect.

Another matter to be mentioned in this connection is the part played by the skilled craftsman in the use of imperfect materials, as already referred to in the introduction to this paper. This consideration is probably of greater importance than those already named and indeed, in itself, may be a sufficient explanation of the bridging of the gap between the required and the attained degree of optical homogeneity of refracting media.

\section{Causes of Nonuniformity in Refractivity of Glass}

A priori, variations in chemical composition would seem to be the causes of nonuniform index in "well annealed" optical glass, and such views predominated until it was shown [9] conclusively that the index differences in good annealed optical glass can be greatly reduced by reannealings in which more care is taken to have uniformity of temperature in the glass during the annealing processes. In short, it was discovered that thermally induced inhomogeneity could be present without appreciable accompanying birefringence. Assuming that the chemical composition varies in glass so as to produce somewhat vaguely delimited regions (relatively very large as compared to striae) having different indices, then a blank made from such glass can pass all usual tests and be regarded as homogeneous, unless prism tests of index are made from several peripheral portions of the blank.

A similar condition of potentially dangerous and vaguely delimited inhomogeneity can occur because of temperature gradients that exist in the furnace during annealing. Prior to demands for large components for wind-tunnel optics, there appeared no necessity for using extreme care to eliminate these small temperature gradients. Consequently, it is impossible to say to what further extent the uniformity of glass may be improved, or to what extent a residual variation in chemical composition may exist. The part that is thermal in origin can be erased by still greater care in heat treatment, but that of chemical nature probably presents more serious problems. Reports made on the degree of homogeneity of glass without quantitative appreciation of the direct effects of nonuniform heat treatment are of little value for estimating the attainable degree oî uniformity.

\section{Stress Birefringence}

The subject of strain still plays such a prominent part in discussions of annealing that it is necessary, especially in view of the high standards of homogeneity that seem really desirable, to determine some quantitative measures of its contribution to optical heterogeneity. The birefringence that it causes is a commonly used measure of the internal stress producing the strain, and it would seem desirable if possible to continue to use the same indicator as a measure of the lack of homogeneity ${ }^{3}$ thus occasioned.

\footnotetext{
3 For the sake of clearness it seems necessary to observe that it is of course true that any piece of glass in which strain exists is as a whole a balanced system involving both compressions and tensions so that in a certain sense the index changes produced, being of opposite sign, offset each other. But portions of the glass showing definite birefringence of a given sign experience a corresponding change in index, and such portions of the medium may be legitimately considered to the exclusion of other portions, because such procedure enables us to arrive at an estimate of the lack of homogeneity arising or existing in situ in a large optical component.
} 
The use of Neumann's [10] equations is so well known through the work of Pockels [11], that it is unnecessary to give details of the derivation of the equation for expressing the absolute birefringence, $\left(n_{y}-n_{z}\right)$, in terms of the unidirectional thrust, $P$, and certain constants of the glass. As written by Adams and Williamson [12], it is

$$
\left(n_{y}-n_{z}\right)=\frac{n P}{E}(1+\sigma)\left(\frac{q}{v}-\frac{p}{v}\right)
$$

where the constants of the glass are, E, Young's modulus; $\sigma$, Poisson's ratio; $n$, the index of refraction of the unstrained medium in which the velocity of light is $v$; whereas $p$ and $q$ are coefficients determined experimentally. The subscripts $y$ and $z$ appended to the symbol $n$ represent the indices of the medium for light vibrating in planes parallel and perpendicular, respectively, to the direction of the thrust. This equation was obtained by subtraction, from the equations

$$
\left(n_{n}-n\right)=\frac{n P}{E}\left(-2 \sigma \frac{p}{v}+\frac{q}{v}\right)
$$

and

$$
\left(n_{z}-n\right)=\frac{n P}{E}\left[(1-\sigma) \frac{p}{v}-\sigma \frac{q}{v}\right] .
$$

From eq 1 it is evident that when $p=q$ (which, as Pockels showed, occurs with a flint glass containing about $74 \%$ of $\mathrm{PbO}$ ), no amount of unidirectional pressure is sufficient to produce birefringence. But eq 2 and 3 indicate that in such cases there are, nevertheless, certain index changes (unless $\sigma=0.50$ as for an incompressible fluid, or unless $p=q=0)$. Thus it might seem that Pockels' work should be extended before it can safely be assumed that no other glasses exist that are exceptional in this respect. Certainly it seems evident that birefringence is not in general a thoroughly reliable quantitative measure of index changes produced, either by external pressure or internal stress, the sensitivity of such a test decreasing as it is used on glasses whose compositions approach that of a glass having the exceptional property described.

On the other hand, the measurements of birefringence that have been made by Pockels, Filon [13], and Adams and Williamson [14] on a combined total of 24 optical glasses, show a small and regular variation giving little or no indication of the existence of other compositions having the remarkable properties referred to in the case of the 74-percent-PbO glass. Circumstances thus seem to warrant a continuation of the use of birefringence as a qualitative measure of strain in all of the usual types of glass, except high-index flints included within the approximate limits of index 1.8 to 1.9 , and also its extension to the qualitative measurement of the change in index so produced.

By the use of eq 2 and 3, and assuming reasonable upper limits of tensile strength, it is possible to arrive at some idea concerning the order of magnitude of the index changes that internal stresses may cause. In this way, it can be shown that strain could never have produced more than a fraction of the lack of homogeneity, which has been commonly attributed thereto. Also, it may be seen that the present practice of annealing to reduce birefringence to 5 or $10 \mathrm{~m} \mu / \mathrm{cm}$ is ample [15] (with possible exception of indices 1.8 to 1.9) for the elimination of strain effects on index to a degree consistent with the most exacting of previous requirements of optical uniformity. On the other hand, in the absence of extensive tests on the character and magnitude of surface deformation that may result from the presence and gradual release of strain, it cannot definitely be said that the present standards of stress removal are unnecessarily high.

\section{Chemical Heterogeneity}

After publication of Eckert's work [16], it ap-peared that the intrinsic heterogeneities in optical glass were not necessarily as large as previously supposed. Briefly, the result bearing on the present question of intrabland homogeneity was his intramelt spread of only $2 \times 10^{-5}$ in refractive index within each of three melts. This was soon confirmed, for glass of European origin, by the reannealing of certain lens blanks at this $\mathrm{Bu}-$ reau [9]. According to this report, the chemical intramelt index variations among six random samples from a single melt were confined to a few units of the sixth decimal place. Such a high degree of chemical homogeneity, if found in all types of optical glass, will mean that with proper heat treatment the ideal of homogeneous refracting media of standardized index is attainable.

Some interesting evidence bearing on this subject is to be obtained from use of the interference method of testing a glass melt for uniformity as described by Dalladay and Twyman [17]. Their 
procedure is in reality a test of the chemical heterogeneity only because, as pointed out by Lebedeff [18], the reannealing of the composite block insures the canceling of physiochemical effects. Consequently the high degree of homogeneity that these investigators found is also encouraging evidence of possible chemical uniformity.

Precise data on the index uniformity of samples taken from pots of optical glass were determined at this Bureau [19] on six types of optical glass. In each case 10 prisms were cut after recording their relative positions and linear separations in two or three dimensions. After precise index measurements by the method of minimum deviation, it was possible to compute approximate index gradients in the original larger pieces of each type of glass from which the individual prisms were taken. These gradients ranged in value from 6 to $49 \times 10^{-6}$ per decimeter but, in general, it was found that the gradients were roughly proportional to the changes that can be produced in the refractive indices of these glasses by heat treatment. Moreover, in each case, as might be inferred from the facts as stated, two prisms of each melt having maximum difference in index were annealed together in the same furnace, and their index differences were thereby very materially reduced. Since the original differences were themselves in the fifth decimal place, it was indicated that the chemically caused variations must indeed be small in the sixth decimal over distances of a decimeter in the glass.

Recently, 10 2-in. cubes of borosilicate glass were annealed at this Bureau and, for each of the three orthogonal directions, the "average" index along each of 24 paths through $2 \mathrm{in}$. of glass was compared with that for a similar average central path through the face centers. This was done on a Twyman-Green interferometer by the method that Twyman [20] has recommended for thin plates. The surfaces are very good but need not be perfect, and the near parallelism is adjusted to give a convenient number of fringes. By transmitted light each fringe delineates the path corresponding to constancy of $2(n-1) t$ whereas, by shielding both mirrors, one can see fringes of constancy of $2 n t$. In other words, in these two cases the fringes are different functions of $n$, the average index of refraction of the glass along the glass path that is traveled. If a cube of homo- geneous glass ( $\Delta n=0$ from path to path through a perfect cube) is slightly thicker at one edge, then the number of fringes seen by reflection and by transmission must be in the ratio $n / n-1$. Any other value of this ratio shows at once that the glass is inhomogeneous. Both $\Delta t$ and $\Delta n$ may be evaluated and the corresponding contours plotted with respect to any arbitrarily selected path. The index differences found for each of the three faces of one of the cubes were \pm 2.5 , \pm 3 , and $\pm 4 \times 10^{-6}$, but the average for 27 faces of the 9 other cubes was only $\pm 1 \times 10^{-6}$.

\section{Temperature Gradients in Annealing Furances}

The desirability of uniformity of heat conditions in annealing was stressed at this Bureau even before the true nature of the thermal effects was fully realized [21]. For the annealing of optical components for use in wind tunnels, the necessity of uniform temperature conditions must now be stressed with multiple emphasis. At the same time there is one fortunate alleviating feature. Whereas, when thinking in terms of strain alone, relatively slight fluctuations were considered important, especially during cooling through certain temperature ranges, now [22], it is plain that, considering temperatures in the annealing range, only an integrated effect is of highest importance for optical uniformity. Consequently it is suggested that the best way of investigating effective deleterious furnace gradients is by measurement of the index heterogeneity actually impressed on small prismatic samples of the glass, which can be located at various places in the furnace while annealings are in progress.

It is by means of this method that it is sometimes possible to analyze index data, post mortem, and get useful information concerning the effective temperature gradients that existed in the annealing furnaces that were used. For example, from the data that Eckert published it is possible to infer that furnace temperature differences during his fine annealings were as large as 1 or $3^{\circ} \mathrm{C}$ over the unknown linear distances that may have been involved. Temperature differences of this order, or greater, over distances of 10 to $30 \mathrm{~cm}$ have probably existed during almost all of the best annealings until very recently. Their possible maximum effects can easily be estimated 
from the list of equilibrium temperature coefficients of refractivity that have been determined at this Bureau for 21 optical glasses. They range from 20 to $60 \times 10^{-6}$ for various silica glasses, depending roughly on the mole fractions of nonsilica oxides that are used in their composition. The full sensitivity of glasses to annealing temperature is thus about 10 times as great as their sensitivity to room temperature changes.

Considering borosilicate glass, which is the type most often used for large windows and interferometer plates, equilibrium temperature coefficients of $38,38,50$, and $53 \times 10^{-6}$ per deg $\mathrm{C}$ have been found on samples differing somewhat in chemical composition. If the full potential effects of temperature gradients in glass during the holding period of an annealing should be realized, it is obvious that the steady systematic furnace gradients must, in general, be reduced to a very few hundredths of 1 degree over the linear dimensions of an optical component, if optical glass is to be homogeneous to $1 \times 10^{-6}$ in index. Fortunately, in practice, the full effects are not realized. If the holding temperature is high enough to permit equilibrium during the short holding periods, then it is likely that further changes during the early stages of cooling will ameliorate the inhomogeneity. If the holding temperature is low, the holding time may not be long enough for attainment of the full inhomogeneity corresponding to the temperature gradient. However, very large pieces must be annealed at low holding temperatures, and the period must be long for the proper relaxation of stresses. Thus they will probably experience a larger proportion of the full equilibrium changes in index than is the case for smaller components with which the glass makers have had more experience.

At this Bureau the temperature gradients in annealing furnaces have progressively and successfully been reduced by placing the glass in a closely fitting metal box, which is inclosed in another box or boxes, that is by inserting more and more metal in the furnace between the glass and the heating coils. Large disks or other large components of glass are symmetrically placed with respect to this added metal and with respect to the furnace walls. Alternate layers of conducting and nonconducting materials are particularly advantageous. Copper as a conductor is objectionable because of excessive oxidation. Aluminum has been found very satisfactory but, for some glasses, care must be exercised to keep preheating below the melting point of this metal. The temperature range can be satisfactorily extended by use of certain alloys of aluminum.

The importance of uniform annealing temperature is so great in the successful production of wind-tunnel optics that it may be necessary to provide for slow continuous rotations of the glass or of the furnace in which it is contained. Possibly simultaneous or successive rotations about two axes may be desirable. Certainly a very high degree of temperature uniformity will be necessary if we are to determine just what degree of residual inhomogeneity is chemical in nature. At present we seem to be safe in saying that in much of our properly selected best optical glass the maximum inhomogeneities are small in the sixth-decimal place over distances of several inches.

\section{References}

[1] A. Q. Tool and E. E. Hill, Trans. Soc. Glass Tech. 9, 185 (1925).

[2] Lord Rayleigh, Phil. Mag. 8, 409 (1879).

[3] S. Chalmers, Trans. Opt. Soc. (London) 18, 192 (1917).

[4] L. C. Martin, Trans. Opt. Soc. (London) 23, 88 (1921).

[5] A. E. Conrady, Mon. Notices Roy. Astr. Soc. 79, 591 (1919).

[6] Dictionary of Applied Physics IV, 222 (Macmillan and Co., Ltd., London, 1923).

[7] F. L. O. Wadsworth, Astro. Phys. J. 16, 267 (1902); 1\%, 1 (1903).

[8] A. E. Conrady, Mon. Notices Roy. Astr. Soc. 79, 582 (1919).

[9] L. W. Tilton, A. N. Finn, and A. Q. Tool, BS Sci. Pap. 22, 719 (1928) S572.

[10] F. Neumann, Ann. Physik 54, 449 (1841).

[11] F. Pockels, Ann. Physik [4] y, 745 (1902); 9, 221 (1902); 11, 652 (1903).

[12] L. H. Adams and F. D. Williamson, J. Wash. Acad. Sci. 9, No. 20, 618 (1919).

[13] L. N. G. Filon, Cambridge Phil. Soc. Proc. 11, 478 (1902); 12, 55 (1903).

[14] L. H. Adams and F. D. Williams, J. Opt. Soc. Am. 4, 217 (1920). 
[15] L. W. Tilton, J. Research NBS 14, 413 (1935) RP776.

[16] Fritz Eckert, Z. Tech. Phys. 7, 282 to 287 (1926).

[17] A. Dalladay and F. Twyman, Jr., Soc. Glass Tech. 5, 325 (1921).

[18] A. A. Lebedeff, Die Glas Industrie 35, 9 (1929).

[19] L. W. Tilton, J. Wash. Acad. Sci. 20, 121 (1929).

[20] F. Twyman, Prism and lens making, p. 136 (Adam Hilger, London).
[21] A. Q. Tool and J. Valasek, BS Sci. Pap. 15, 562 (1920) S358.

[22] A. Q. Tool, L. W. Tilton, and J. B. Saunders, J. Research NBS 38, 519 (1947) RP1793.

Washington, September 3, 1948. 